\title{
Epileptic Encephalopathy
}

\author{
Nicola Specchio, ${ }^{1}$ Marina Trivisano, ${ }^{2}$ Rod C. Scott,, 4 and Colin Ferrie ${ }^{5}$ \\ ${ }^{1}$ Department of Neuroscience, Bambino Gesù Children's Hospital, IRCCS, Piazza S, Onforio 4, 00165 Rome, Italy \\ ${ }^{2}$ Clinic of Nervous System Diseases, University of Foggia, Foggia, Italy \\ ${ }^{3}$ Department of Neurology, Geisel School of Medicine at Dartmouth, Lebanon, NH, USA \\ ${ }^{4}$ UCL Institute of Child Health, London, UK \\ ${ }^{5}$ Department of Paediatric Neurology, Leeds General Infirmary, Leeds LS1 3EX, UK
}

Correspondence should be addressed to Nicola Specchio, nicola.specchio@gmail.com

Received 19 December 2012; Accepted 19 December 2012

Copyright (C) 2012 Nicola Specchio et al. This is an open access article distributed under the Creative Commons Attribution License, which permits unrestricted use, distribution, and reproduction in any medium, provided the original work is properly cited.

Epileptic encephalopathies are conditions in which epileptic activity itself is postulated to contribute to severe cognitive and behavioural impairments above and beyond what might be expected from the underlying pathology alone.

The term has been used in two ways: (1) as a generic classification term for epilepsies with severe cognitive and behavioural outcomes and (2) as a pathophysiological process. We argue that the term is not synonymous with "severe epileptic syndrome". Epileptic encephalopathy can complicate many different epileptic conditions. In some it is an almost constant feature (e.g., Lennox-Gastaut syndrome), in others expected but not invariable (e.g., West syndrome) whilst in yet others it may or may not occur with almost equal frequency (e.g., Doose syndrome). Epileptic encephalopathy very occasionally complicates otherwise benign epilepsies (e.g., benign rolandic epilepsy). Epileptic encephalopathy is a dynamic condition that may persist over time causing increasingly severe functional effects or else it may improve and remit, either spontaneously or with treatment which suppresses the proposed causative epileptic activity.

Fundamental to the concept of epileptic encephalopathy is that the cognitive and other problems which characterise it result from epileptic activity, rather than being a consequence of any underling cerebral pathology (whether genetic, structural, metabolic inflammatory, etc.) which may itself be causing the epilepsy. However, all spontaneous epileptic phenomena in humans are a function of some kind of brain disease (genetic through to structural) and therefore it is likely that all cognitive impairments in children with epilepsy are at least in part a function of aetiology.
When a brain disorder gives rise to both epilepsy and cognitive and behavioural problems, it should not be classed as an epileptic encephalopathy, but rather as an "epileptogenic encephalopathy". The crucial difference being that the cognitive and behavioural problems are not a consequence of the epilepsy, but of the cerebral pathology. However, in epileptogenic encephalopathies seizures may aggravate cognitive and behavioural problems and thus treatment of seizures may improve outcomes.

Some disorders, mostly genetically determined and characterised by the onset of seizures in the first months of life and with developmental stagnation are best considered as "early onset epilepsies with encephalopathy" rather than epileptic encephalopathy. Both the epilepsy and the encephalopathy appear to be symptoms of a known or unknown genetic defect and there is no evidence that epileptic activity is primarily responsible for the developmental stagnation. Examples of this include disorders associated with CDKL5, SCN1A, and PCDH19 mutations.

This special issue contains five papers on epileptic encephalopathy. The paper by S. Khan and R. Al Baradie is a review of the clinical and neurophysiological features of the most common epileptic encephalopathies occurring in infancy and childhood whilst the paper by Z. Kural and A. F. Ozer examines current approaches to treatment of childhood and adult epileptic encephalopathies. They emphasise that an appropriate differential diagnosis of epileptic seizures and "infraclinical epileptic discharges" are crucial to the management of seizures, epileptic discharges and the related cognitive regression. 
E. Sheppard and S. Lippé examine the possible consequences of prolonged and recurrent status epilepticus on cognitive development. They correlate the occurrence and severity of epileptic encephalopathy with risk factors such as aetiology, duration and frequency and age at onset of seizures. D. Brazzo et al. explore a specific type of epileptic encephalopathy (status epilepticus during sleep), including the pathophysiological mechanisms underling the condition and the insights which may come form the use of new and emerging imaging techniques.

In the paper J. Ricardo-Garcell et al. examine associated risk factors that can contribute to the cognitive impairment in patients with epileptic encephalopathy. Prematurity and severe asphyxia were often responsible of neurodevelopmental delay. In such cases distinguishing the role of epilepsy from the underlying brain pathology in causing the encephalopathy is very difficult.

Nicola Specchio

Marina Trivisano

Rod C. Scott

Colin Ferrie 


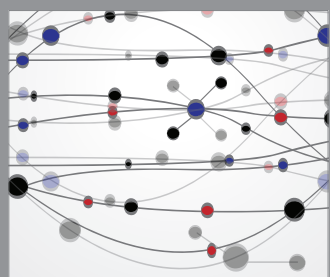

The Scientific World Journal
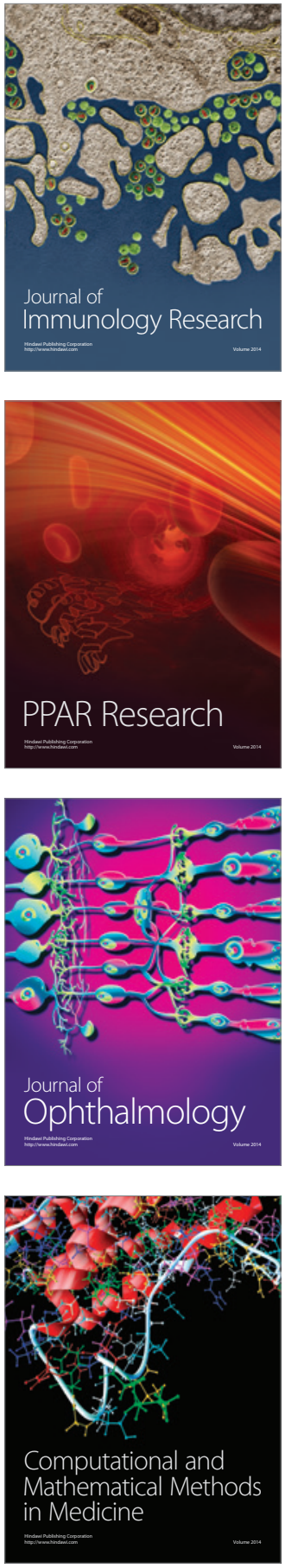

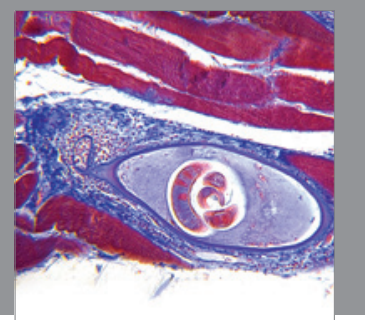

Gastroenterology

Research and Practice
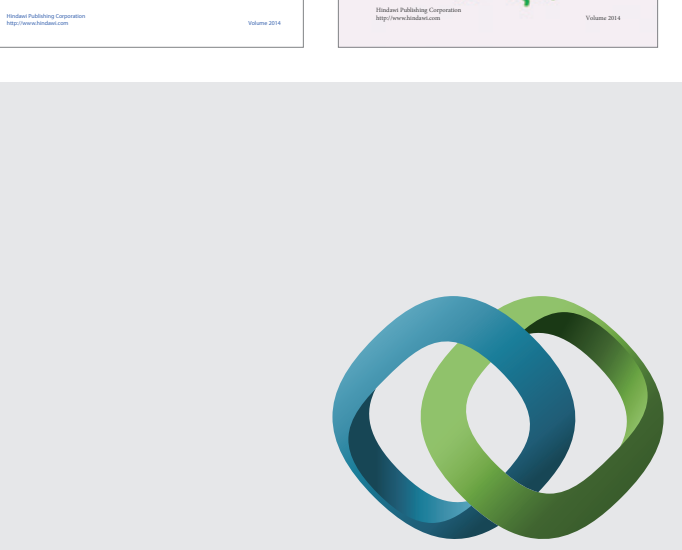

\section{Hindawi}

Submit your manuscripts at

http://www.hindawi.com
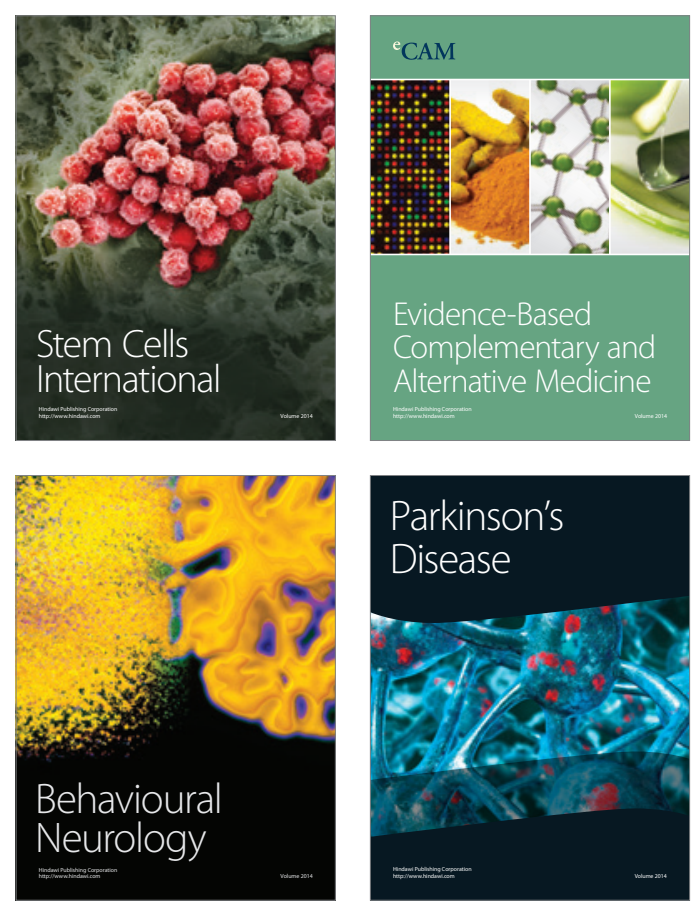

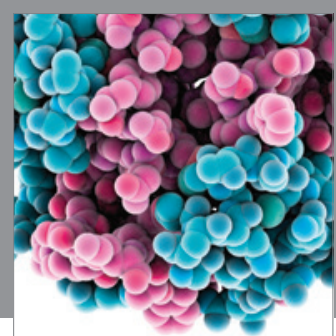

Journal of
Diabetes Research

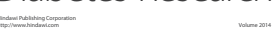

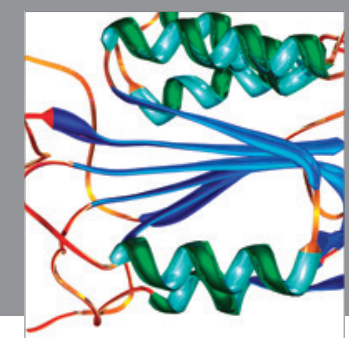

Disease Markers
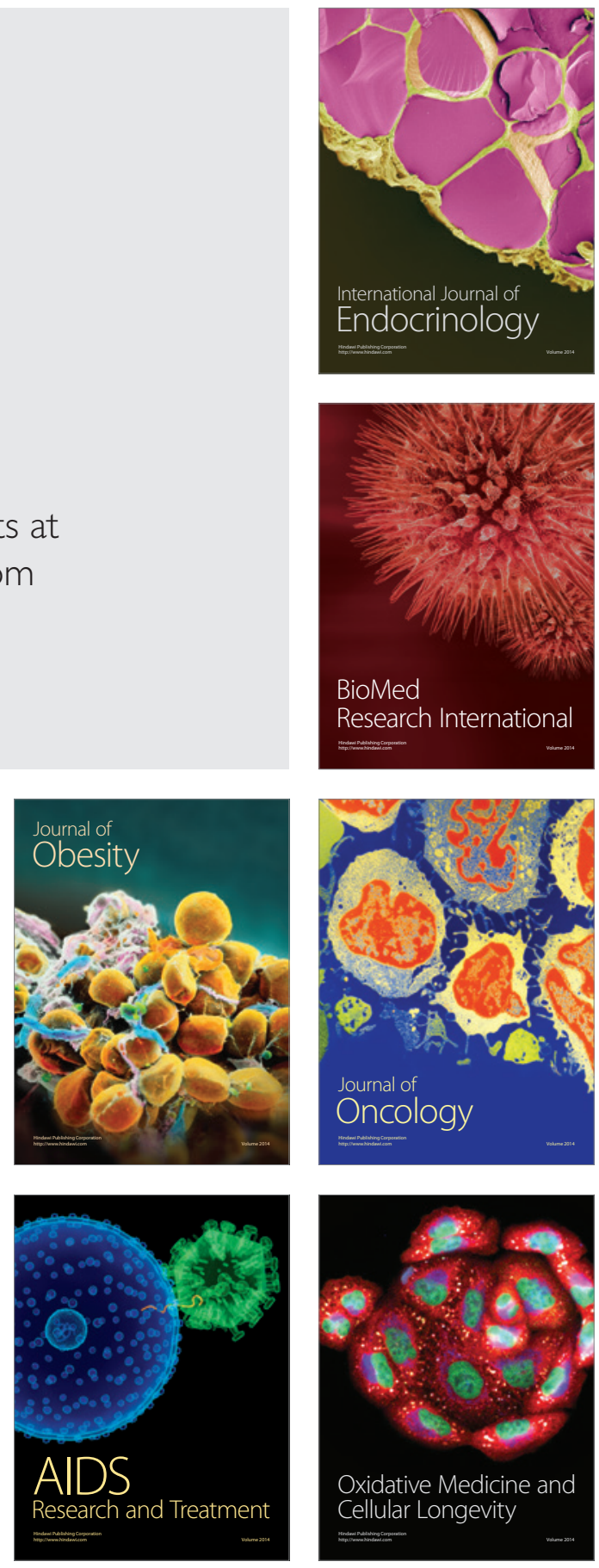